\title{
O MÉDICO VETERINÁRIO NA ATENÇÃO BÁSICA À SAÚDE
}

The veterinarian in Primary Health Care

El veterinario en Atención Primaria

\author{
Daniele Rosa Xavier ${ }^{*}$, Guilherme N. L. do Nascimento ${ }^{2}$ \\ ${ }^{1}$ Doutoranda, Faculdade de Saúde Pública, Curso de Doutorado em Saúde Pública, \\ Universidade de São Paulo, São Paulo, Brasil.
}

${ }^{2}$ Laboratório de Ciências Básicas e da Saúde - LaCiBS, Curso de Nutrição, Universidade Federal do Tocantins, Palmas/TO, Brasil.

*Correspondência: Faculdade de Saúde Pública, Universidade de São Paulo, Av. Dr. Arnaldo, 715, Pinheiros, São Paulo/SP, Brasil.CEP:03178-200.E-mail danni_vet@yahoo.com.br.

\section{RESUMO}

Considerando a atuação do médico veterinário em atividades de saúde pública, e a sua inclusão nas equipes multidisciplinares do Núcleo de Saúde da Família, a presente pesquisa teve como objetivo apresentar os desafios deste profissional da saúde, bem como sua atuação e importância nesta área. Para elaboração deste trabalho foi realizado um estudo bibliográfico sobre o tema em questão. O amplo campo profissional contempla desde a gestão e o planejamento em saúde até a mais tradicionalmente conhecida vigilância epidemiológica e sanitária. Atuam ainda na prevenção, controle e erradicação das doenças veiculadas por diversos fatores, entre os quais podemos citar: origem alimentar, parasitária, infecciosas, entre outros. Assim, o papel estratégico do médico veterinário na prevenção e no controle de enfermidades deve ser constante a ponto de permitir a partilha de experiências entre outros profissionais da área da saúde a fim de salvaguardar a vida dos animais e principalmente da população humana.

Palavras-chave: Saúde Pública, Médicos Veterinários, Estratégia Saúde da Família.

\section{ABSTRACT}

Considering the role of the veterinarian in public health activities, and their inclusion in multidisciplinary teams from the Family Health Center, this research aimed to present the challenges of this health professional and his role and importance in this area. For preparation of this paper, we conducted a bibliographic study on the subject in question. The broad professional field comprises from management and health planning to the more traditionally known epidemiological surveillance and environmental health. They act even in prevention, control and eradication of diseases transmitted by several factors, among which include: food-borne, parasitic, infectious, among others. Thus, the strategic role of the veterinarian in the prevention and control of diseases should be constant enough to allow the sharing of experiences among other health professionals in order to safeguard the lives of animals and especially of the human population.

Keywords: Public Health, Veterinarians, Family Health Strategy.

\section{RESUMEN}

Teniendo en cuenta el papel del veterinario en las actividades de salud pública, y su inclusión en equipos multidisciplinarios del Centro de Salud de la Familia, esta investigación tuvo como objetivo presentar los retos del profesional de la salud y su función e importancia en esta área. Para la preparación de este trabajo se llevó a cabo un estudio de la literatura sobre el tema en cuestión. El amplio campo profesional comprende de gestión y planificación de la salud a la vigilancia epidemiológica más tradicionalmente conocido. Actúan también en la prevención, control y erradicación de las enfermedades de transmisión por varios factores, entre los que se 
incluyen: transmitidas por los alimentos, parasitarias, infecciosas, entre otros. Por lo tanto, el papel estratégico del veterinario en la prevención y control de enfermedades debe ser lo suficientemente constante para permitir el intercambio de experiencias entre los otros profesionales de la salud con el fin de salvaguardar la vida de los animales y en especial de la población humana.

Descriptores: Salud Pública, Veterinarios, Estrategia de Salud Familiar.

\section{INTRODUÇÃO}

A saúde pública veterinária consiste na aplicação do conhecimento do profissional de Medicina Veterinária para a proteção e promoção da saúde humana, sendo incluído como categoria profissional de nível superior para atuação no Conselho Nacional de Saúde através das resoluções $n^{\circ} 038 / 1993$ e no 287/1998 (BRASIL, 1993, 1998).

O Programa Saúde da Família (PSF), composto por equipes multidisciplinares, tem funcionado como uma estratégia de reorientação do modelo assistencial no âmbito das unidades de saúde (CRMV-SC, 2008; BRASIL, 2011; PAIM et al., 2011). Atua em ações de promoção da saúde, prevenção, recuperação, reabilitação de doenças e agravos mais frequentes, e na manutenção de saúde de uma comunidade, com número definido de famílias localizadas em uma área geograficamente delimitada (CRMV-SC, 2008; STORTI, 2012).

Portanto, a entrada do médico veterinário na atenção básica à saúde é imperativo no campo da prevenção e controle das doenças transmissíveis a humanos, nos serviços de saúde pública em geral e nas estratégias de ação em equipes de Saúde da Família (COSTA, 2011; BARBOSA, 2014).

Logo, se objetivou com este trabalho caracterizar a importância e campo de atuação deste profissional nas ações de saúde, principalmente na atenção básica.

\section{MATERIAIS E MÉTODOS}

Trata-se de um estudo teórico sobre o campo de atuação, a inclusão e importância do médico veterinário na saúde pública e participação no Programa de Saúde da Família.

O levantamento bibliográfico foi realizado nas bases de dados: Scielo, Lilacs, Ibecs, Medline e Google Acadêmico, com os descritores: Estratégias de Saúde da Família; Inserção do médico veterinário na saúde pública; Núcleo de Atenção Básica à Saúde.

Foram incluídos neste trabalho artigos publicados em periódicos científicos, resoluções, normas e leis pertinentes ao assunto em estudo. $\mathrm{O}$ período de tempo para realização do levantamento destas informações ocorreu nos meses de janeiro a dezembro de 2016.

\section{RESULTADOS E DISCUSSÃO}

Papel do médico veterinário e áreas de atuação na saúde pública

A profissão de médico veterinário foi criada no Brasil em 1918 e regulamentada pelo decreto-lei $n^{\circ}$ 23.133, de 09 de setembro de 1933 (BRASIL, 1933).

Tendo como áreas de atuação: $i$ ) prevenção e cura de enfermidades de diversas espécies animais, ii) produção e inspeção de produtos de origem animal (leite, carne, ovos e mel), iii) defesa sanitária animal, iv) saúde pública, v) ensino técnico e superior, vi) pesquisa, vii) extensão rural e viii) preservação ambiental e ecológica. Participa também do intercâmbio de informações entre a pesquisa médica veterinária e a humana; estudo sobre substâncias tóxicas e venenos provenientes dos animais considerados peçonhentos; supervisão da criação de animais de experimentação; estabelecimento de 
interligação e cooperação entre as organizações de saúde pública e Veterinária com outras unidades relacionadas com animais (Fig.1) (WORLD HEALTH ORGANIZATION, 2002; PFUETZENREITER, 2003; BÜRGER, 2010).

Anteriormente vislumbrado como um profissional voltado exclusivamente para o atendimento aos animais (BÜRGER, 2010), o médico veterinário teve seu papel ampliado dentro do contexto da saúde da família, atuando no processo de administração, planejamento e coordenação de programas em saúde coletiva nas diversas esferas de governo federal, estadual e municipal (GUIMARÃES et al., 2010; COSTA, 2011).

Figura 1. Representação esquemática das áreas de atuação do médico veterinário.

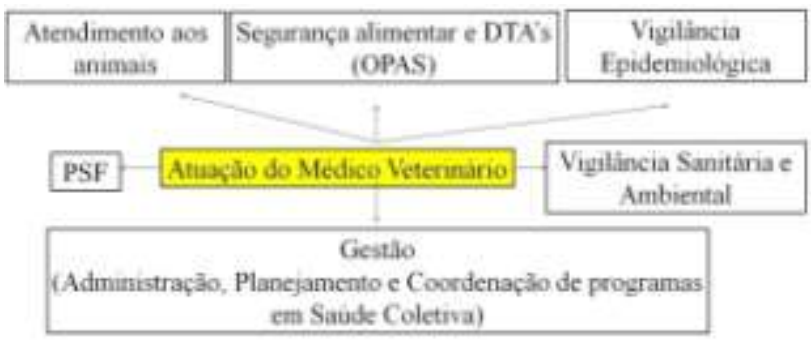

$\mathrm{Na}$ saúde pública, sua participação é compreendida em dois períodos distintos. Inicialmente, final do século XIX ao início do século $\mathrm{XX}$, atuando em atividades relativas à higiene $\mathrm{e}$ proteção dos alimentos, como, por exemplo, na indústria de carne, e pouca, mas significativa, participação administrativa em programas correlacionados, como Controle e Vigilância em Zoonoses (SCHWABE, 1984; PFUETZENREITER et al., 2004; TAFFAREL, 2014). Posteriormente, contribuiu como propulsor da saúde coletiva, através do desempenho de atividades nas áreas técnicoadministrativas (SCHWABE, 1984; PFUETZENREITER et al., 2004).
No âmbito internacional, fundou-se em 1902, e formalmente estabelecida em 1924, a Organização Pan-Americana de Saúde (OPAS) (COSTA, 2011), que forneceu as bases políticas e técnicas para o desenvolvimento e fortalecimento da saúde pública veterinária nas Américas (ARÂMBULO III, 2008), mediante o auxílio destes profissionais no planejamento, na definição de políticas, alocação de recursos, e fornecimento de orientação técnica e apoio ao pessoal operacional, além da preocupação com a segurança alimentar e doenças transmitidas por alimentos (ARÂMBULO III, 2008; COSTA, 2011).

Assim, a saúde pública veterinária contribui para o bem-estar físico, mental e social dos seres humanos por meio da compreensão e aplicação dos conhecimentos da Medicina Veterinária, visando proteger e promover a saúde humana, através de vínculos com a agricultura, alimentação, saúde animal, meio ambiente e educação (PFUETZENREITER e ZYLBERSZTAJN, 2008).

O ensino da saúde pública em Medicina Veterinária

No Brasil, houve uma rápida expansão dos cursos de Medicina Veterinária nos últimos anos, devido à implementação de novas vagas pelo governo e execução pelo Ministério da Educação (OLIVEIRA FILHO et al., 2009).

Segundo dados disponibilizados pelo Ministério da Educação, no ano de 2015, havia aproximadamente 234 cursos de graduação em Medicina Veterinária em atividade no Brasil (BRASIL, 2015).

Em relação ao ensino da saúde pública veterinária, que compreende as práticas preventivas e de Inspeção e Tecnologia de Produtos de Origem Animal (POA), apresentavam em 2010, carga horária 
de 422,62 horas, sendo menos privilegiada quando comparada às áreas de Clínica Veterinária e de Zootecnia e Produção (BÜRGER, 2010).

No entanto, as atividades da Medicina Veterinária em saúde pública iniciaram-se no século XIX, na indústria da carne, com Robert von Ostertag (Alemanha) e Daniel E. Salmon (Estados Unidos) (ORGANIZACIÓN PANAMERICANA DE LA SALUD, 1975). Todavia, o termo saúde pública veterinária só foi utilizado oficialmente em 1946, durante um encontro onde a Organização Mundial da Saúde (OMS) direcionou os conhecimentos de Medicina Veterinária para a proteção e promoção da saúde humana, bem como no ensino nas escolas de veterinária, sendo posteriormente traduzido em diversas línguas (WORLD HEALTH ORGANIZATION, 1951; PFUETZENREITER, 2003; TAFFAREL, 2014).

A saúde pública é um dever do Estado, mas cabe uma ação conjunta de todos os segmentos da sociedade na prevenção, proteção e promoção da saúde da população, aliado aos recursos técnicos e participação das autoridades sanitárias, agrícolas e os profissionais envolvidos, quer na clínica ou na pesquisa, quer no setor público ou no setor privado (MEDITSCH, 2006).

Com isto o ensino tem sofrido mudanças e os profissionais desta área passaram a ter maior inserção nas discussões e práticas da saúde pública, focando suas atividades simultaneamente aos animais e consequentemente aos seres humanos.

\section{Programa de Saúde da Família e Núcleo de Apoio à Saúde da Família}

De acordo com Nunes (1993), a construção do Programa Saúde da Família (PSF) no Brasil aconteceu em um contexto de redemocratização e reforma dos serviços públicos. O PSF surgiu como estratégia para a melhoria do atendimento prestado à população, tendo como foco a prevenção voltada à orientação dos usuários (SILVA e DALMASO, 2004), através da criação e adaptação de estratégias de atendimento no âmbito do Sistema Único de Saúde (SUS), com vistas à busca de racionalização e melhoria da qualidade dos serviços públicos, à necessidade de maior equidade, cidadania, garantia de direitos e bem estar integral da população como um todo (SENNA e COHEN, 2002).

Essa facilitação do acesso da população aos serviços básicos de saúde, dispensando a esta serviços de qualidade, bem como profissionais capacitados, só foi possível a partir da implantação do PSF e consequentemente descentralização da atenção da saúde (CARVALHO, 1995).

A inclusão do médico veterinário na área da saúde data do ano de 1991, porém somente efetivada pela Resolução do Conselho Nacional de Saúde (CNS) $n^{\circ}$ 38, de 04 de fevereiro de 1993 (BRASIL, 1993), pois esta categoria profissional estava relacionada somente à área de Ciências Agrárias e não à da Saúde (MEDITSCH, 2006).

Conforme entendimento do Ministério da Saúde, sob portarias $n^{\circ} 154$, de 24 de janeiro de 2008 (BRASIL, 2008) e $n^{\circ} 2.488$, de 21 de outubro de 2011 (BRASIL, 2011), os Núcleos de Apoio à Saúde da Família (NASF) foram criados com o intuito de aumentar a área de atuação das equipes de saúde atuantes nos diversos programas do governo como a Estratégia Saúde da Família (ESF) e Atenção Primária à Saúde (APS) (ANDRADE et al., 2012), devendo atender a diretrizes preestabelecidas e relacionadas à APS, com a ação interdisciplinar dos profissionais e intersetorial com promoção de ações 
voltadas para a educação permanente tanto dos profissionais quanto da população, visando à integralidade e humanização popular (SENNA e COHEN, 2002).

Inclusão do médico veterinário na Atenção Básica à Saúde da Família

A Atenção Básica à Saúde, segundo Ministério da Saúde (2011), é caracterizada por um conjunto de ações de saúde, no âmbito individual e coletivo, que envolve a promoção e a proteção da saúde em todas as suas vertentes, com múltipla atuação de profissionais da saúde.

A partir da resolução $n^{\circ} 287$, de 8/10/1998 do CNS (BRASIL, 1998) e pela Portaria Interministerial $n^{\text {o } 45,12 / 01 / 2007 ~ d o s ~ M i n i s t e ́ r i o s ~ d a ~ E d u c a c ̧ a ̃ o ~ e ~ d a ~}$ Saúde (BRASIL, 2007), que tratam da questão da atuação multidisciplinar no setor da saúde, o médico veterinário passou a fazer parte do corpo de profissionais que atuam no NASF, porém de forma não obrigatória quanto a sua contratação (COSTA, 2011).

Vale ressaltar que a participação do médico veterinário na saúde pública é de suma importância, no que diz respeito à prevenção e minimização dos riscos de exposição dos homens às zoonoses, decorrentes da crescente demanda por alimentos de origem animal, ocasionando o incremento das indústrias zootécnicas e de rebanhos (GERMANO, 1983), além da expansão do mercado pet com aquisição de animais exóticos (aves ornamentais, hamsters, quelônios e serpentes) e deslocamento de animais às feiras e exposições (PFUETZENREITER e ZYLBERSZTAJN, 2008).

No entanto, ainda precisamos avançar no cenário de atuação do médico veterinário na construção de políticas e estratégias de saúde pública no Brasil, tanto pela baixa representatividade desta classe nos serviços de saúde, como pela ausência de articulação social e política destes em Conselhos Municipais de Saúde (NAPOLI, 2011).

O médico veterinário pode atuar de forma multidisciplinar em atividades conjuntas tais como a clínica, em diagnósticos, na inspeção e supervisão de animais e na área da pesquisa, bem como em ações de educação em saúde e na participação nos setores de gestão estratégica na Atenção Básica de estados e municípios.

No entanto, destaca-se que apesar da formalização da composição do médico veterinário no NASF, não há garantia de sua participação no programa em discussão, pois fica a critério do poder executivo dos municípios (prefeituras, secretários de saúde, entre outros) a sua inclusão na equipe, mediante dados epidemiológicos, necessidades locais e haver disponibilidade de recursos financeiros. Com isso, faz-se necessário o conhecimento dos gestores em saúde quanto à importância da participação destes profissionais no processo de decisão e atuação na área da saúde básica.

A difusão dos conhecimentos obtidos nos cursos de graduação, bem como aos provenientes da prática profissional, possibilitará sua atuação também nas áreas administrativas, de planejamento e na coordenação de programas de saúde pública em nível nacional, estadual ou municipal. Esta preocupação com prática da promoção em saúde deve-se aos diversos fatores que tem colaborado para a disseminação de enfermidades, principalmente no que concerne ao contato "íntimo" com os animais de companhia aumentando assim, os riscos de exposição às zoonoses. 


\section{CONCLUSÃO}

A participação do médico veterinário na saúde pública e em Programas de Atenção Básica, como o Núcleo de Saúde da Família é de suma importância para a consolidação da saúde e melhoria dos serviços públicos disponibilizados à população, uma vez que, a saúde envolve vários fatores, sendo necessário o olhar de diferentes profissionais para abordar todas as variáveis e condicionantes da saúde, sendo de responsabilidade do médico veterinário a observação e contribuição em aspectos relacionados à integração homem-animal.

Todos os autores declararam não haver qualquer potencial conflito de interesses referente a este artigo.

\section{REFERÊNCIAS}

ANDRADE, L.M.B.; QUANDT, F.L.; CAMPOS, D.A.; DELZIOVO, C.R.; COELHO, E.B.S.; MORETTI-PIRES, R.O. Análise da implantação dos Núcleos de Apoio à Saúde da Família no interior de Santa Catarina. S\&TS/H\&SC. p.18-31, 2012.

ARAMBULO III, P. Internacional programs and veterinary public health in the Americas - Success, challenges and possibilities. Prev. Vet. Med. v.86, n. 3-4, 2008.

BARBOSA, D.S. A inserção do médico veterinário nos Núcleos de Apoio à Saúde da Família (NASF): novos caminhos de atuação na saúde pública. JMPHC. v.5, n.1, p.1-3, 2014.

BRASIL. Decreto $\mathrm{n}^{\mathrm{o}} 23.133$ de 9 de setembro de 1933. Regula o exercício da profissão veterinária no Brasil e dá outras providências.

BRASIL. Conselho Nacional de Saúde. Resolução ${ }^{\circ}$ 038 de 04 de fevereiro de 1993. Inclui os cursos de Biologia, Medicina Veterinária e Serviço Social entre os cursos relacionados no item $n^{\circ} 3$ da Resolução CNS n 017 do Conselho Nacional de Saúde, de 28 de novembro de 1991.

BRASIL. Conselho Nacional de Saúde. Resolução ${ }^{\circ}$ 287 , de 18 de outubro de 1998 . Resolve sobre a inclusão de categorias profissionais de saúde de nível superior para atuação no Conselho Nacional de Saúde.

BRASIL. Ministério da Educação e da Saúde. Portaria Interministerial $n^{\circ} 45,12 / 01 / 2007$. Dispõe sobre a Residência Multiprofissional em Saúde e a Residência em Área Profissional da Saúde e institui a Comissão Nacional de Residência Multiprofissional em Saúde. DOU, Edição $n^{\circ}$ 10, 15/01/2007.

BRASIL. Ministério da Saúde. Portaria n ${ }^{\circ}$ 154, de 24 de janeiro de 2008. Cria os Núcleos de Apoio à Saúde da Família - NASF. DOU, Brasília, DF, no 43, 4 março de 2008.

BRASIL. Ministério da Saúde. Portaria $n^{\circ} 2.488$, de 21 de outubro de 2011. Aprova a Política Nacional de Atenção Básica, estabelecendo a revisão de diretrizes e normas para a organização da Atenção Básica, para a Estratégia Saúde da Família (ESF) e o Programa de Agentes Comunitários de Saúde (PACS).

BRASIL. Ministério da Educação. Sistema e-MEC, $2015 . \quad$ Disponível em: $<$ http://emec.mec.gov.br/emec/consultacadastro/detalhescurso/d96957f455f6405d14c6542552b0f6eb/MzIy/9f 1aa921d96ca1df24a34474cc171f61/Mzg=>. Acesso em: 15 dez. 2016.

BÜRGER, K.P. O ensino da Saúde Pública Veterinária nos cursos de graduação em Medicina Veterinária do estado de São Paulo. 2010. Tese de Doutorado em Medicina Veterinária Preventiva. Universidade Estadual Paulista - UNESP/Jaboticabal; 2010.

CARVALHO, A.I. Conselhos de Saúde no Brasil: participação cidadã e controle social. Rio de Janeiro, Ibam; 1995.

COSTA, H.X. A importância do médico veterinário no contexto de saúde pública. In: Seminário Programa de Pós-Graduação em Ciência Animal da Escola de Veterinária da Universidade Federal de Goiás, Goiânia: UFG, 2011.

CRMV-SC. Conselho Regional de Medicina Veterinária do Estado de Santa Catarina. Médicos Veterinários no Programa Saúde da Família. Informe, n.2, 2008. 
GERMANO, P.M.L. Saúde pública em Medicina Veterinária. Hig. Aliment. v.2, n.3, 1983.

GUIMARÃES, F.F.; BAPTISTA, A.A.S.; MACHADO, G.P.; LANGONI, H. Ações da vigilância epidemiológica e sanitária nos programas de controle de zoonoses. Vet. Zootec. v.17, n.2, p.151-162, 2010.

MEDITSCH, R.G.M. O médico veterinário na construção da saúde pública: um estudo sobre o papel do profissional da clínica de pequenos animais em Florianópolis, Santa Catarina. CFMV, Brasília/DF, n.38, Ano XII, 2006.

NAPOLI, L.O. Contexto do médico veterinário na saúde pública contemporânea. CRMV/PR, 2011. Disponível em: <http://www.crmvpr.org.br/?p=imprensa/artigo_detalhes\&id=81>.

Acesso em: 25 jan. 2017.

NUNES, T.C.M. Educação continuada: uma proposta para a área de Epidemiologia no SUS. Inf. Epidemiol. 1993.

OLIVEIRA FILHO, B.D.; SANTOS, F.L.; MONDADORI, R.G. O ensino da Medicina Veterinária: realidade atual e perspectivas. CFMV, Brasília/DF, n.46, Ano XV, 2009.

ORGANIZACIÓN PANAMERICANA DE LA SALUD. A competency-based curriculum for veterinary public health and preventive medicine. Washington: Paho/WHO; 1975.

PAIM, J.; TRAVASSOS, C.; ALMEIDA, C.; BAHIA, L.; MACINKO, J. O sistema de saúde brasileiro: história, avanços e desafios. Lancet, Séries Saúde no Brasil, 2011.

PFUETZENREITER, M.R. O ensino da Medicina Veterinária Preventiva e Saúde Pública nos cursos de Medicina Veterinária - Estudo de caso realizado na Universidade do Estado de Santa Catarina. 2003. Tese de Doutorado em Educação. Universidade do Estado de Santa Catarina - UESC; 2003.

PFUETZENREITER, M.R.; ZYLBERSZTAJN, A.; ÁVILA-PIRES, F.D. Evolução histórica da Medicina Veterinária Preventiva e Saúde Pública. Ciênc. Rural, v.34, n.5, 2004.

PFUETZENREITER, M.R.; ZYLBERSZTAJN, A. Percepções de estudantes, professores e médicos veterinários sobre o ensino da Medicina Veterinária
Preventiva e Saúde Pública. Rev. Ciênc. Agrovet. v.7, n.1, p.75-84, 2008.

SCHWABE, C.W. Veterinary medicine and human health. Baltimore: Williams \& Wilkins; 1984.

SENNA, M.C.M.; COHEN, M.M. Modelo assistencial e estratégia saúde da família no nível local: análise de uma experiência. Ciênc. saúde coletiva, v.7, n.3, p.523-535, 2002.

SILVA, J.A.; DALMASO, A.S.W. Agente Comunitário de Saúde: o ser, o saber, o fazer. Cad. saúde pública. 2004.

STORTI, M.M.T. As diretrizes da educação permanente em saúde nos Núcleos de Apoio à Saúde da Família - NASF. 2012. Monografia de Especialização. Universidade Federal de Santa Catarina - UFSC; 2012.

TAFFAREL, A.C. Aspectos do papel do médico veterinário na Saúde Pública, o ensino curricular e o conceito de One Health: revisão de literatura e considerações. 2014. Monografia. Universidade Federal do Rio Grande do Sul - UFRS; 2014.

WORLD HEALTH ORGANIZATION. Joint WHO/FAO Expert Group on Zoonoses - Report on the First Session, Geneva, n.40; 1951.

WORLD HEALTH ORGANIZATION. Future Trends in Veterinary Public Health: Report of a WHO Study Group, Geneva: WHO Technical Report Series; 2002. 